Storms, E. Use Of A Very Sensitive Seebeck Calorimeter To Study The Pons-Fleischmann And Letts Effects. in Tenth International Conference on Cold Fusion. 2003. Cambridge, MA: LENR-CANR.org. This paper was presented at the 10th International Conference on Cold Fusion. It may be different from the version published by World Scientific, Inc (2003) in the official Proceedings of the conference.

\title{
Use Of A Very Sensitive Seebeck Calorimeter To Study The Pons- Fleischmann And Letts Effects
}

\author{
EDMUND STORMS \\ Energy K.Systems \\ Storms2@ix.netcom.com
}

\begin{abstract}
Characteristics of a commercial Seebeck calorimeter are described. This very stable instrument is applied to a study of the Pons-Fleischmann effect using a palladium anode and a platinum cathode. The use of a laser to stimulate anomalous heat production (the Letts effect) is also described. Positive results were obtained for both effects and these reveal important aspects of the nuclear-active-environment.
\end{abstract}

\section{INTRODUCTION}

Dennis Letts ${ }^{1}$ described success in making significant anomalous energy while irradiating a Pons-Fleischmann (P-F) cathode with laser light near $680 \mathrm{~nm}$ using 30-35 $\mathrm{mW}$ of applied laser power. This study was done using a laser supplied by Dennis Letts and with his collaboration. During his study, the cathode was palladium that had been subjected to a complex series of treatments and then plated with gold. The attempt at replication described in this paper is based on the assumption that the active surface consists of deposited nanocrystals that are not uniquely related to the nature of the substrate material. Therefore, surfaces were applied using other, less complex methods including deposition of gold on untreated palladium outside of the calorimeter and deposition of palladium on platinum or on copper, both in situ.

This study initially used a stirred isoperibolic calorimeter. While this method could accurately measure changes in power produced by the laser, it was not stable enough to reveal existence of anomalous power in the absence of laser light, i.e. the Pons-Fleischmann effect. Therefore, a Seebeck calorimeter was modified to allow absolute power to be measured in the presence and absence of laser light.

A Seebeck calorimeter creates a voltage that is proportional to heat passing through its walls. This voltage is converted to a known heat flux by using a calibration based on generating a known amount of power within the Seebeck box. Each time the Seebeck was opened and changes were made in the cell contents, the calorimeter was recalibrated using a Joule heater located within the cell. Fans are present within the Seebeck enclosure to uniformly distribute heat generated by the cell. Excess power (EP) is calculated according to

$$
E P=A+B^{*} V+C^{*} V^{2}-V_{c}^{*} I_{c}-V_{f}^{*} I_{f}
$$

where $\mathrm{V}$ is the Seebeck voltage, $\mathrm{V}_{\mathrm{c}}$ is the voltage between the anode and cathode, $\mathrm{I}_{\mathrm{c}}$ is the current passing through the cell, $\mathrm{V}_{\mathrm{f}}$ is the voltage applied to the fans, $\mathrm{I}_{\mathrm{f}}$ is the current passing through the fans, and A, B, C, are constants obtained from a calibration. An example of a typical 
calibration is shown in Fig. 1. Being a steady-state device, power values are obtained only after all variables have become constant, in this case after $100 \mathrm{~min}$.

\section{EXPERIMENTAL}

\section{Isoperibolic Calorimeter:}

The first study was made using a stirred, jacketed isoperibolic calorimeter (Fig. 1) in which temperature was measured within the electrolyte at three locations (Fig 2) and

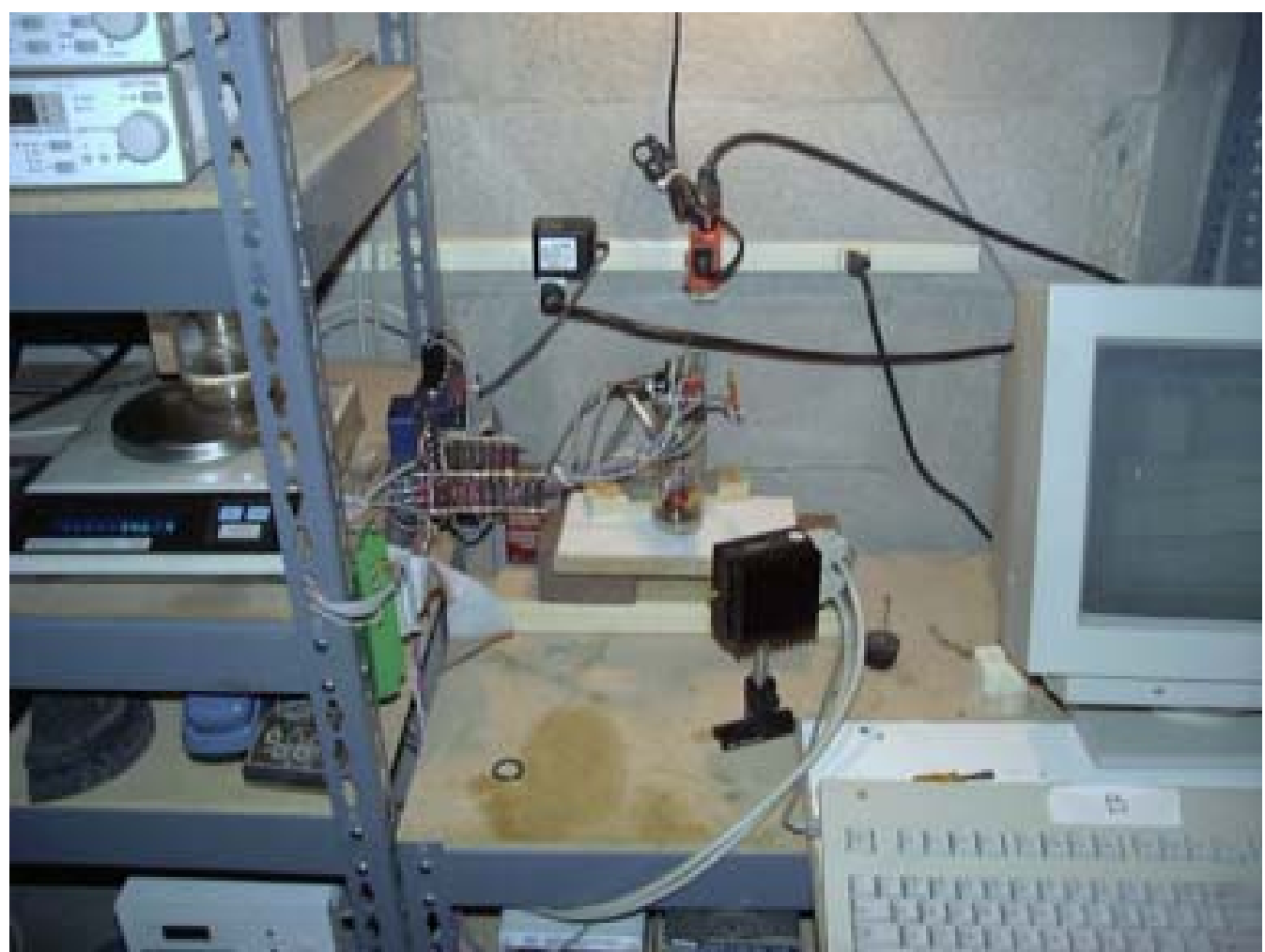

FIGURE 1. Picture of isoperibolic calorimeter before addition of stirrer.

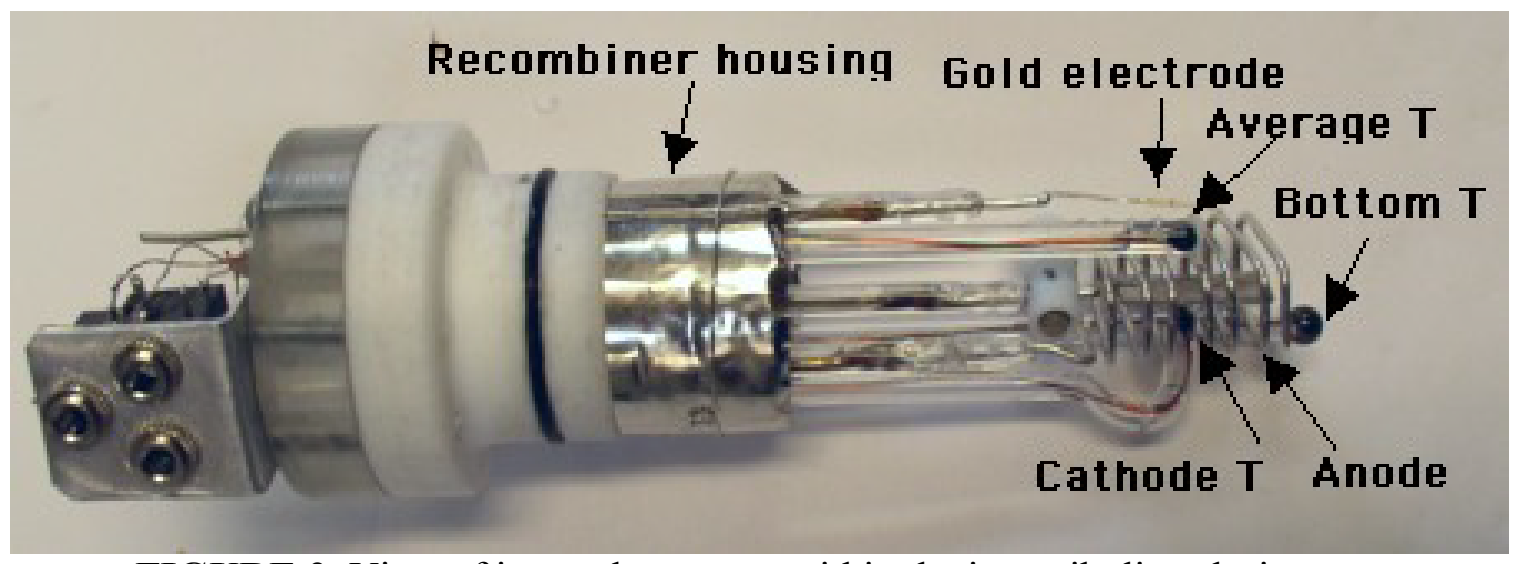

FIGURE 2. View of internal structure within the isoperibolic calorimeter. 


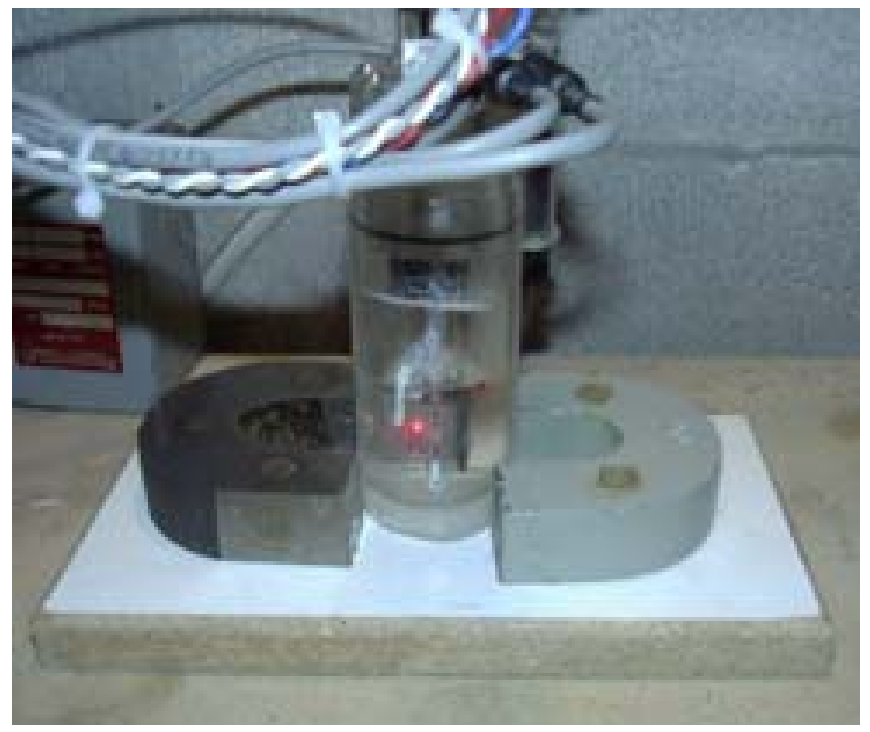

FIGURE 3. Location of magnetics

where cooling water exited the surrounding jacket. A stirring rate of $150 \mathrm{rpm}$ was chosen to minimize thermal gradients between the three internal thermistors. Changes in power production were determined by measuring changes in the temperature difference between a thermistor located within the electrolyte (Average T, Fig. 2) and at the cooling water exit. The calorimeter was calibrated using a "dead" platinum cathode. Relative accuracy, i.e. changes occurring within an hour, was $\pm 5 \mathrm{~mW}$, while long-term accuracy was about $\pm 100 \mathrm{~mW}$. This accuracy allowed the Letts effect to be measured with confidence, but the P-F effect could not. Consequently, the study was transferred to a Seebeck calorimeter where greater accuracy and stability could be obtained.

Effect of applying a magnetic field of 250-300 Gauss to the cathode was explored (Fig. 3 ), as taught by Letts. The presence of the field and its orientation had a significant effect on the calibration constant.

The sample used in this study was prepared by rolling a $1 \mathrm{~mm}$ thick Pd sheet to $0.33 \mathrm{~mm}$ thickness, etching in Aqua Regia, and plating with gold. The surface was gold colored and consisted of a forest of dendrites when viewed using an optical microscope at 1000x.

\section{Seebeck Calorimeter:}

The Seebeck calorimeter was purchased from Thermonetics, Inc. and has a 7"x7"x7" active volume, as can be seen in Fig. 4. Although the calorimeter box is water cooled by pipes attached to the Seebeck plates, the Seebeck voltage is, nevertheless, sensitive to changes in room temperature. Therefore, the Seebeck box is placed in another box in which the temperature is held to within $\pm 0.02^{\circ}$ of the cooling water temperature $\left(20^{\circ} \mathrm{C}\right)$. A study of how airflow within the box changes the calibration constants shows that the walls of the Seebeck box are not uniform in their sensitivity to heat flow. Based on trial and error, three $12 \mathrm{~V}$ squirrel-cage fans were eventually found to improve distribution of heat to the walls so that the entire system can reach thermal 


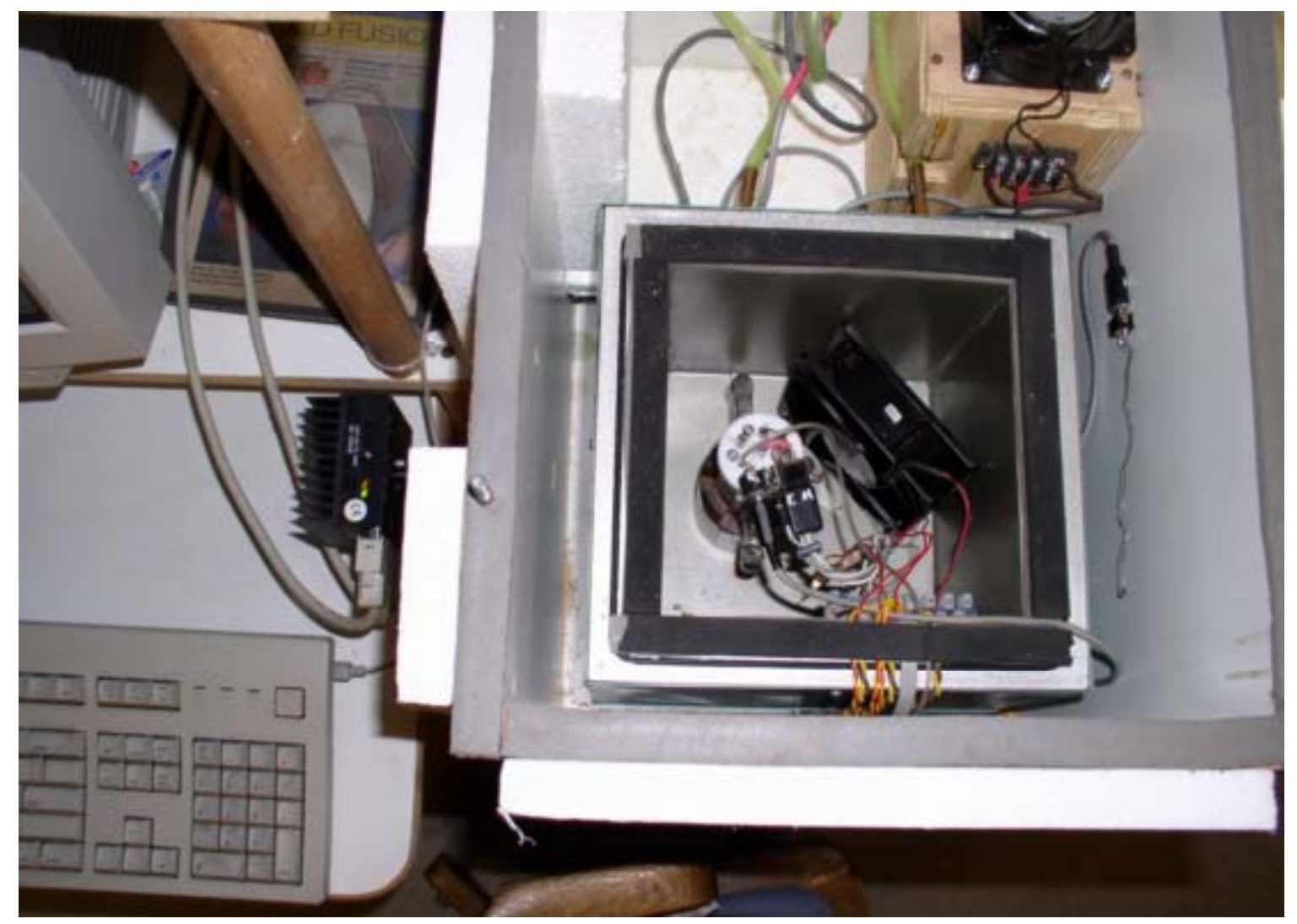

FIGURE 4. Interior view of the Seebeck calorimeter. The Seebeck box is located in a wooden enclosure that is held at $\pm 0.02^{\circ} \mathrm{C}$. Only one fan is used in this design.

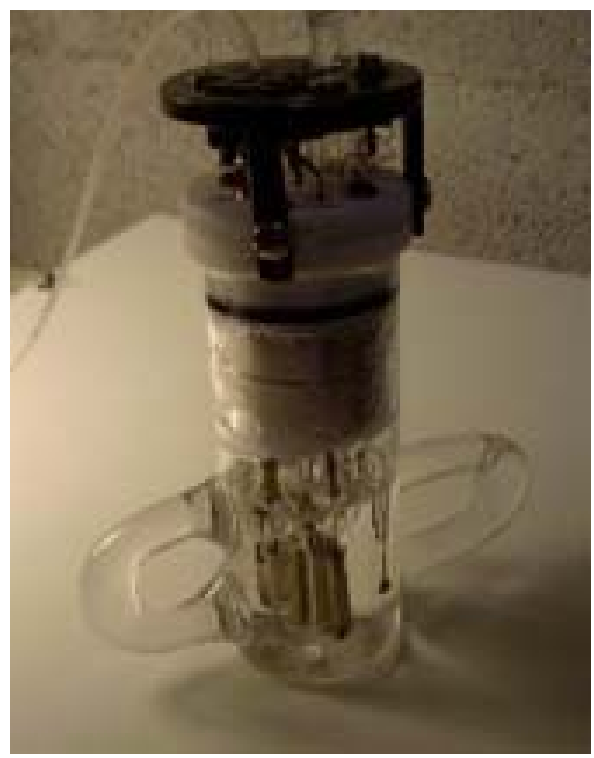

FIGURE 5. Picture of cell used in the Seebeck calorimeter. The ears are used to help heat leave the cell. 
equilibrium more quickly and be more stable. These fans introduce a fixed but measured power of 5.3 watts into the box.

The calorimeter is calibrated before each study by passing measured power through a resistor made from platinum wire. Initially, this wire was exposed to the electrolyte within the cell. Later, a glass enclosed resistance wire was used. This power is measured at the calorimeter boundary using the same data acquisition channels, resistors, and power supply as used when power is applied during electrolysis. As a result, many errors cancel. A National Instruments PCI-6035E data acquisition board controlled by LabView and a Power Macintosh are used while running at 1000 data values/sec. Measured values are found to be independent of data acquisition rate. Time dependent variations are reduced by averaging up to 30,000 values for critical variables.

The cell is made of Pyrex and contains only Teflon, platinum, palladium, $40 \mathrm{ml}$ of 0.10 or $0.19 \mathrm{~mol} \mathrm{Li} / 1000 \mathrm{ml} \mathrm{LiOD}$ electrolyte, and a recombiner consisting of Pt on carbon cloth. A picture is shown in Fig. 5. The recombiner is well isolated from the electrolyte to prevent chemical attack. The electrolyte was made by mixing Cambridge Isotopes, $99.9 \% \mathrm{D}_{2} \mathrm{O}(0.9$ $\mu$ mho) with a suitable amount of LiD. A small gold counter electrode is also present that can be used to measure the referenced open-circuit-voltage or to apply gold to the cathode. Initially, the anode was made of platinum mesh. However, this did not produce consistent success. Later, the anode was switched to self-supporting palladium wire $(99.95 \% \mathrm{Pd})$ that was wrapped around the cathode so as to be equidistant with a $5 \mathrm{~mm}$ gap. One of several metals $(\sim 0.7 \mathrm{~cm} \mathrm{x} \sim 1.5 \mathrm{~cm})$ to which various treatments are applied is used as the cathode. The cathode lead wire $(\mathrm{Pt})$ is attached to the external platinum wire by a Teflon clamp, thereby allowing quick change.

The cell is gas tight and communicates with an oil reservoir. Oil from this reservoir is weighed on a balance $( \pm 0.01 \mathrm{~g})$ to indicate whether gas is leaving or entering the cell. No gas was found to leave or enter the cell except that caused by changes in temperature of the cell.

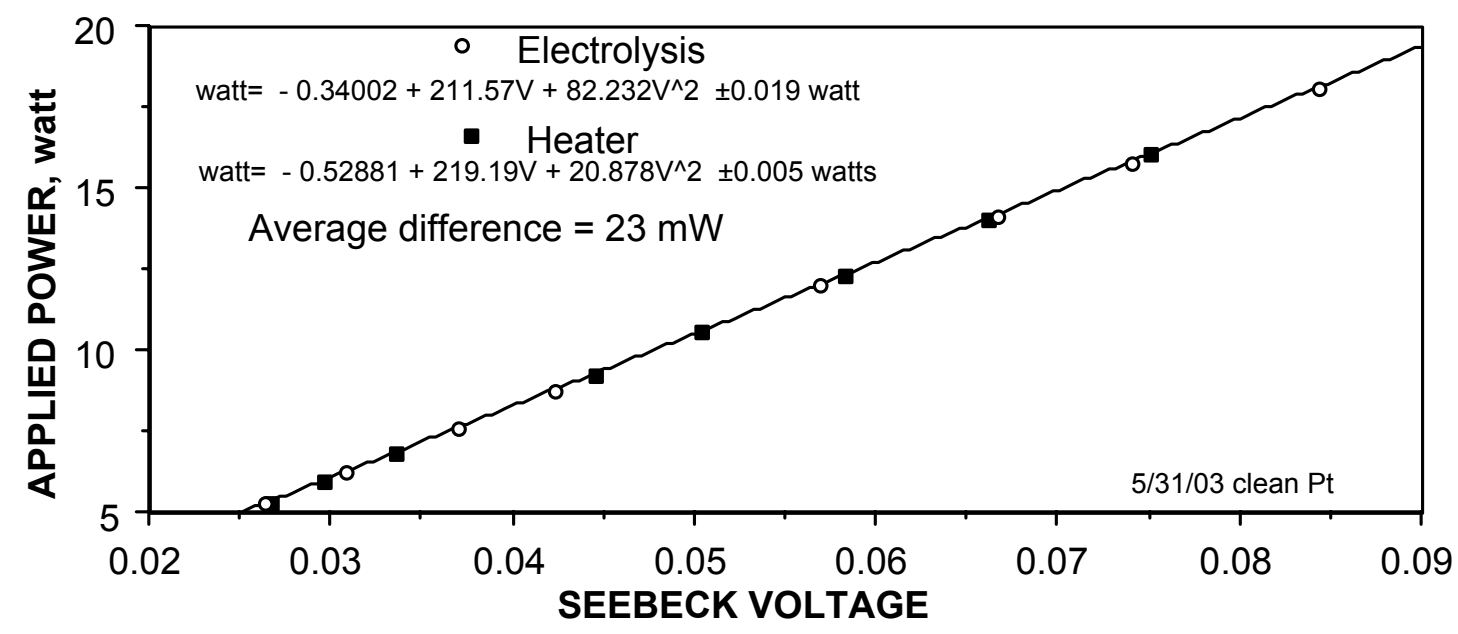

FIGURE 6. Seebeck voltage vs applied power generated by the Joule heater and by electrolysis during calibration while using a clean, "dead" Pt cathode.

The same electrode design, data acquisition equipment, and method to test for gas loss were used during the initial isoperibolic study.

A temperature and current controlled OPTIMA laser diode control was used with a laser having a controlled wavelength between $680-686 \mathrm{~nm}$ and an output power of $30-35 \mathrm{~mW}$. This 
was located outside of the Seebeck box and its constant-temperature enclosure, with laser light entering through an uncovered 3/4" hole. This laser was also used during the initial isoperibolic study.

\section{ERROR}

The maximum absolute error for the Seebeck calorimeter is \pm 0.023 watt, based on comparing the difference between electrolysis using a clean Pt cathode and the internal Joule heater, an example of which is shown in Fig. 6. This means that variations from zero of $\pm 23 \mathrm{~mW}$ are not considered to be abnormal. The maximum random error is $\pm 0.2 \%$ of applied power based on scatter between many points taken under constant conditions. The average deviation between the 9 points taken for the average calibration is $\pm 15 \mathrm{~mW}$. Calibrations made hundreds hours apart and after the cell had been previously opened have a standard deviation from the average of $\pm 25 \mathrm{~mW}$. This is an indication of excellent stability.

The voltage during electrolysis at the highest applied current contains a nonsymmetrical AC component, the value of which is averaged by a $2200 \mu \mathrm{fd}$ capacitor connected across the voltage measuring circuit. The maximum amount of power that can be missed by the measuring circuit is about $25 \mathrm{~mW}$ at $1 \mathrm{~A}$ of applied current, with proportionally less at lower applied current. However, this error is inconsistent with the positive bias of $23 \mathrm{~mW}$ (see Fig. 6) found during electrolysis of a "dead" electrode compared to the heater that has a lower AC component. Nevertheless, as long as each study produces the same constant AC component, the effect is not important to the interpretation of these studies.

The Seebeck voltage is known to $\pm 10 \mu \mathrm{V}$. The major factors that can affect this value are the stability of cooling water temperature and the stability of fan power. Changes in both can destabilize the calorimeter causing uncertain results. Cooling water temperature is constant to $\pm 0.04^{\circ} \mathrm{C}$ over the full range of applied power and fan power has a variation from the mean of $\pm 25 \mathrm{~mW}$, caused mainly by changes in the temperature within the Seebeck box. However, fan power is measured at each point and added to power being generated within the box so that this uncertainty is reduced to $\pm 5 \mathrm{~mW}$.

To avoid changes in convection currents within the box, the cell is returned to exactly the same location each time using indexing pins. No gas leaves the cell as indicated by the nearly constant weight of oil in the oil reservoir.

Temperature is measured within the cell, within the Seebeck box, and at the cooling water outlet using linear thermistors. These thermistors are calibration against a standard to within $\pm 0.04^{\circ}$ and are sensitive to changes of $\pm 0.001^{\circ}$. Temperature is only used to monitor the stability of the system.

\section{RESULTS \\ Isoperibolic Method:}

An example of the Letts effect using the isoperibolic calorimeter is shown in Fig. 7 where laser power is changed between zero and its maximum of $30-35 \mathrm{~mW}$ in steps while a fixed current is applied to a gold-coated palladium cathode. The effect of the laser light increases with time and with the amount of applied laser power. The maximum amount of laser power actually entering the cell was previously determined to be $25 \mathrm{~mW}$. The missing $5-10 \mathrm{~mW}$ is proposed to be reflected off the glass wall of the cell. Excess power eventually grew to be about 10 times applied laser power. 
This calorimeter reached steady state after only 6 minutes, allowing such studies to be made. The resulting excess power (EP) values from this and a similar study are compared to the applied laser power in Fig. 8. No critical onset power is apparent and the effect shows signs of saturating at higher applied power.

This study, during which many examples of the effect were obtained, was deemed a successful replication. However, the magnet used by Letts is found to be unnecessary and the effect appears to be independent of laser wavelength over the range that is available (680-686 $\mathrm{nm})$, contrary to what Letts found.

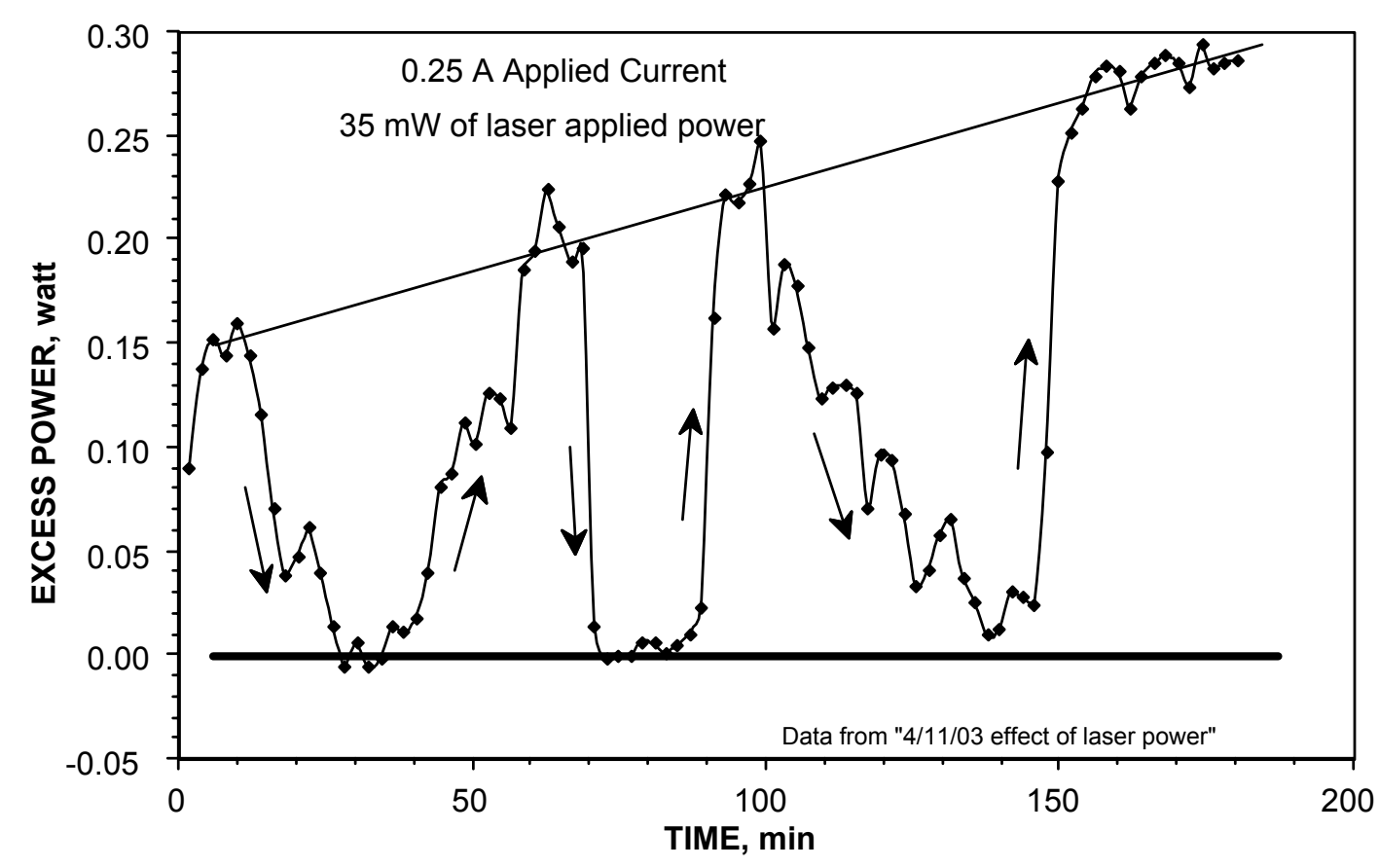

FIGURE 7. Laser power applied to a gold covered Pd cathode using an isoperibolic calorimeter. Arrows indicate when laser power is increasing or decreasing.

\section{Seebeck Method:}

During the study involving the Seebeck calorimeter, anomalous energy is measured while two different procedures were used. For the first, applied current is changed in steps; going first up in current and then going down to give a total of about 9 points. The second method consists of taking data as a function of time after the calorimeter has reached equilibrium, generally in 100 minutes. The first procedure is used during calibration using the internal heater. The laser is occasionally applied while a fixed current is applied to a "dead" cathode to determine the amount of laser energy entering the calorimeter. Anomalous power generated by the laser is obtained by taking the difference between the measured power before and after application of the laser, and then subtracting the amount of power added to the cell by the laser itself. 


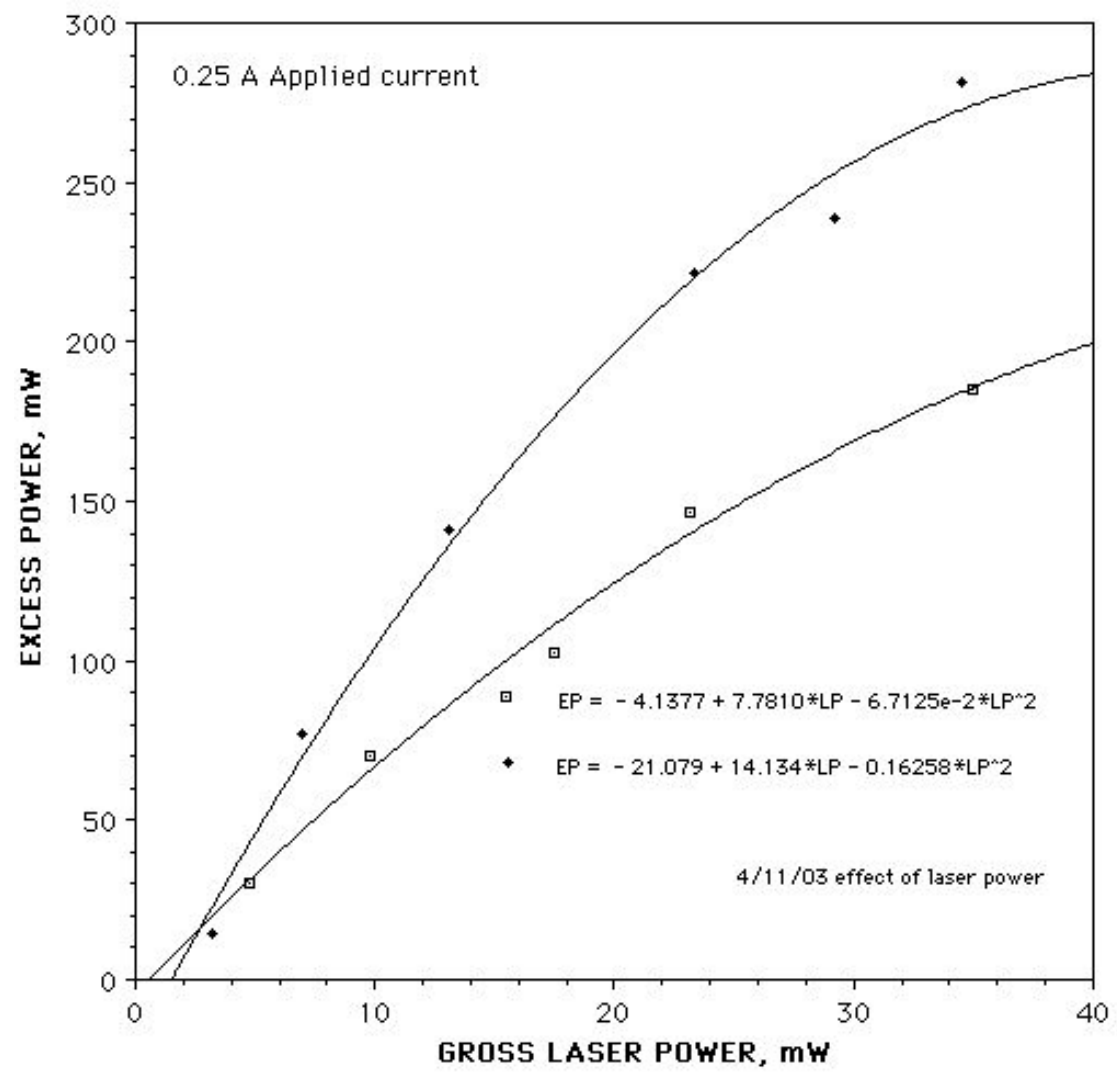

FIGURE 8. Two examples showing the effect of applied laser power on the resulting excess power using a Pd cathode coated with gold.

The first task is to calibrate the calorimeter using the internal Joule heater and by electrolysis using a dead Pt cathode. The platinum cathode is always dead at first and starts to show gradual heat production only after a few hundred hours of electrolysis, depending on the amount of applied current, as a deposit of Pd from the Pd anode builds up on the surface. Figure 6 shows a typical calibration that is fit best by a second degree polynomial. Notice the good internal consistency between points within each data set and between the two methods, as shown on the figure.

\section{Platinum Cathode:}

An initially clean Pt cathode that had been exposed to electrolytic current using a Pd anode is studied using the Seebeck. The amount of heat generated by the laser when applied at several cell currents is shown in Fig. 9. Notice that when $0.5 \mathrm{~A}$ of applied power is used, the amount of measured laser power is $25-30 \mathrm{~mW}$, which is superimposed on $140 \mathrm{~mW}$ of P-F power. The amount of measured laser power is consistent with the intrinsic power of the laser and is taken to be the reference power that needs to be subtracted from all other measurements in order to determine the amount of anomalous power. During this run, application of 1 A generated 220 $\mathrm{mW}$ of P-F power while producing $30 \mathrm{~mW}$ over the intrinsic power of the laser when the laser was applied.

The second Pt cathode was exposed to current only during the initial calibration and the results are shown in Fig. 10. While P-F power is small, it apparently slowly increased during the study. At $0.1 \mathrm{~A}$, the laser deposited about $20 \mathrm{~mW}$ into the cell without producing an 
anomalous effect. Increasing the current to 0.2 A caused anomalous laser power of $50 \mathrm{~mW}$, with an unstable behavior. Application of the laser also caused the internal temperature of the cell to increase by about $0.1^{\circ} \mathrm{C}$, similar to the effect seen when the isoperibolic calorimeter was used. Application of 1.1 A to this sample shows an immediate production of P-F heat that slowly increased with time, as shown in Fig. 11. No Letts effect was observed during this time.

After having established that the calorimeter is able to detect power being applied by the laser and detect the resulting excess power caused by the P-F and Letts effects, the next task is to determine how these effects can be changed by changing the nature of the deposited surface. Small amounts of materials that can accelerate transfer of material from the anode were added. The resulting effect is shown in Fig. 12. First of all, the P-F power is reduced by addition of KI and an indication of a maximum in EP is seen when current is increased above $1.3 \mathrm{~A}$. Addition of $\mathrm{LiCl}$ produces a more pronounced

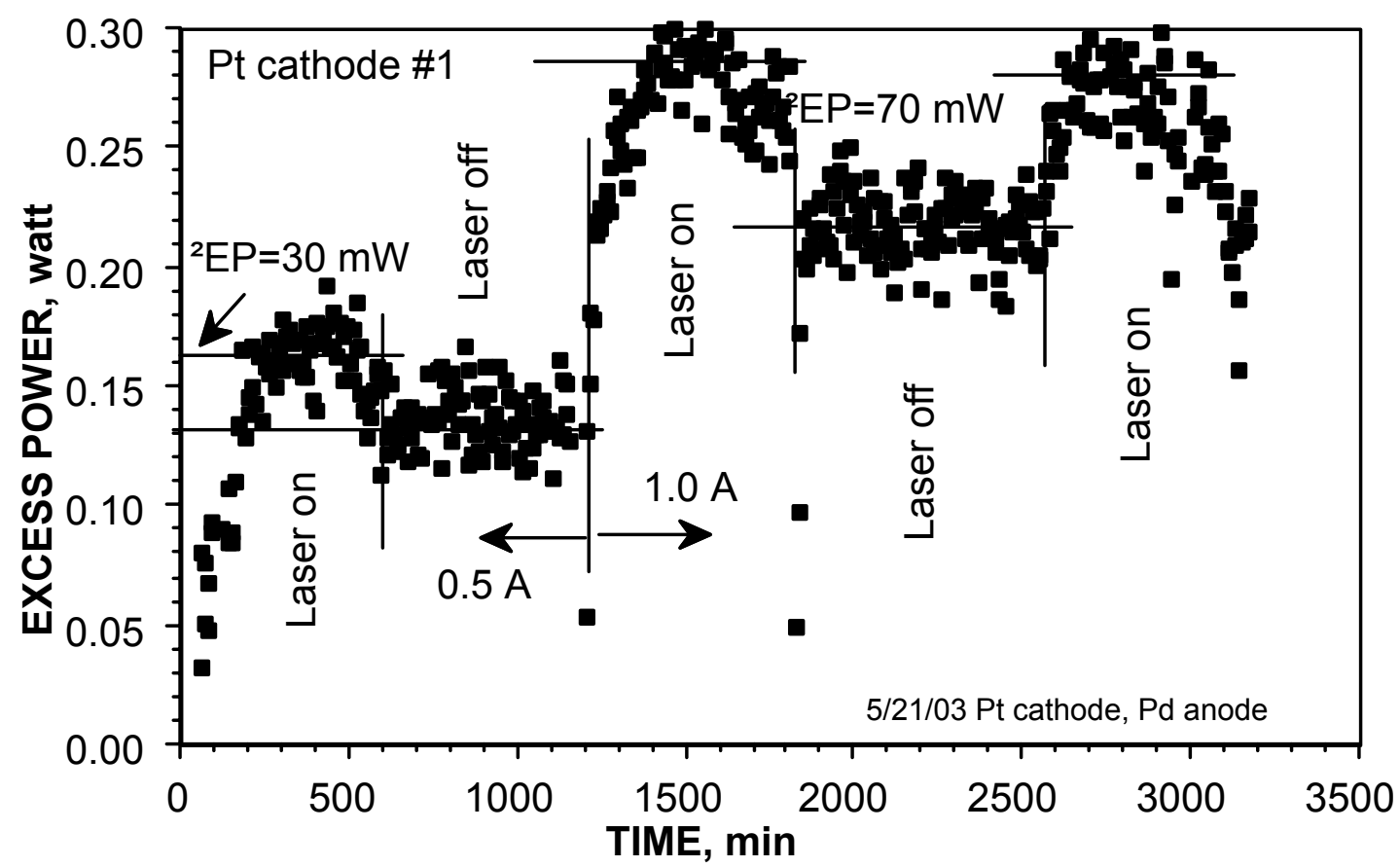

FIGURE 9. Effect of laser light on excess power production at $0.5 \mathrm{~A}$ and $1.0 \mathrm{~A}$ for Pt cathode \#1. This cathode had been exposed to applied current before this measurement. Optimum conditions for reducing data scatter had not yet been achieved during this measurement. 


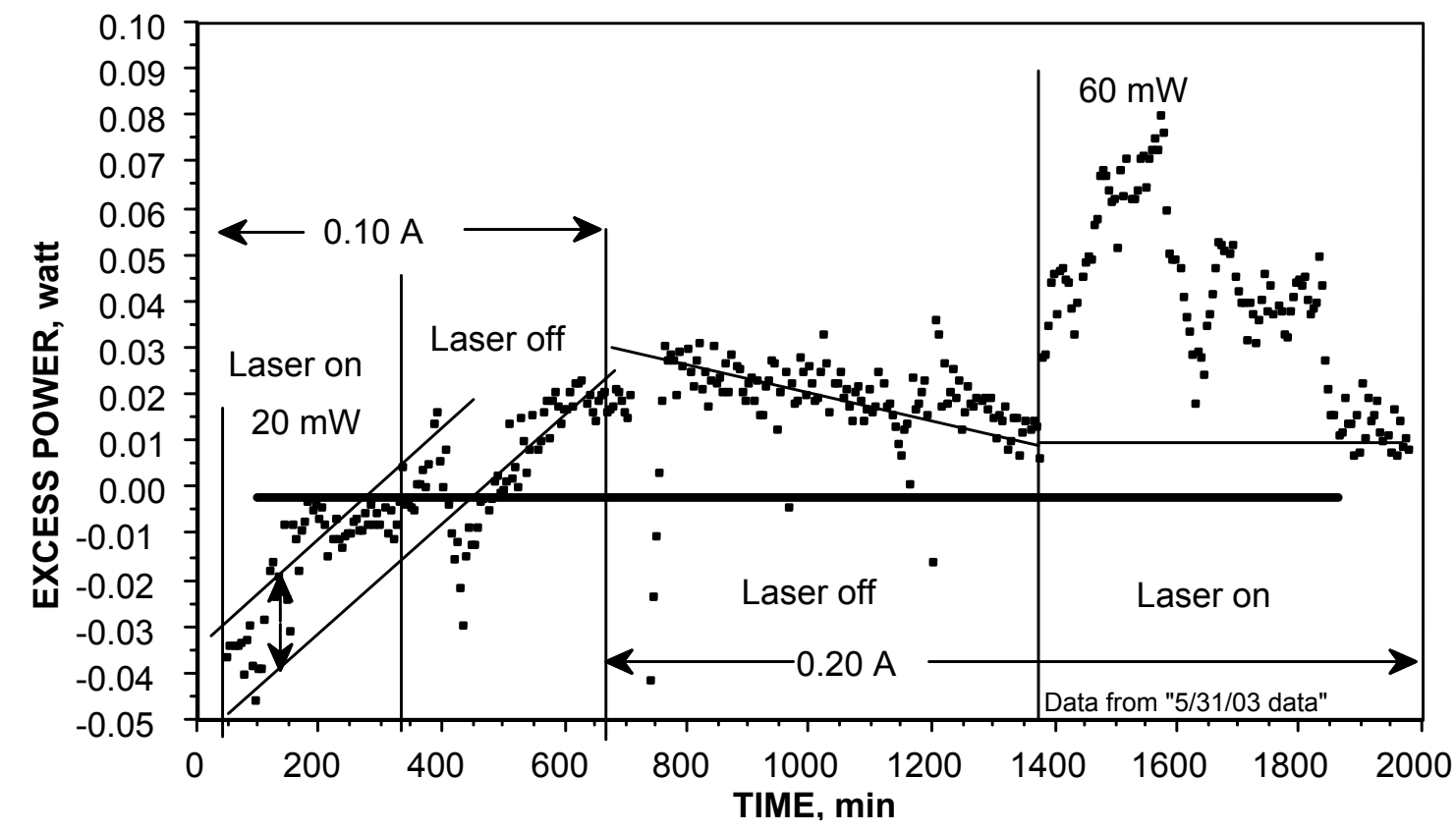

FIGURE 10. Effect of laser light on excess power production at applied currents of $0.1 \mathrm{~A}$ and 0.2 A for Sample \#2. This sample had not been exposed to high current for a significant time. Optimum conditions for data acquisition are used.

maximum and causes the EP to drop to zero at the highest current. No indication of the Letts effect is seen when the laser is applied at $0.5 \mathrm{~A}$ of electrolytic current.

The cathode surface had acquired a black coating that consists of a range of particle sizes with the majority barely visible at 1000x using an optical microscope.

\section{Copper Cathode:}

Having shown that excess power can be produced using a platinum cathode, next a clean copper cathode was treated in a similar fashion using a fresh electrolyte of $0.2 \mathrm{~N}$ LiOD. Figure 13 shows how EP changes with time immediately after electrolysis is started. Application of 1.0 A produces neither Letts nor P-F excess power, although the extra power of $30 \mathrm{~mW}$ added by the laser is clearly visible. However, when current is reduced to $0.5 \mathrm{~A}$, the P-F effect is found to be present, although it is unstable. Initial application of laser energy caused no additional effect, as can be seen at $1830 \mathrm{~min}$. By the time the laser was turned off, the Letts effect had increased to about $130 \mathrm{~mW}$ and the P-F effect had decreased to zero. The line on the figure shows how the P$F$ effect is proposed to change. Further electrolysis caused the P-F effect to increase once again, but eventually both the Letts and P-F effects disappeared before the sample was removed from the calorimeter. The copper surface was covered by a uniform silver-colored layer and had gained $0.0003 \mathrm{~g}$.

\section{DISCUSSION}

If anomalous power (excess power, EP) occurs spontaneously in an electrolytic cell, the result is called the Pons-Fleischmann (P-F) effect. When EP is initiated by exposing the cathode to laser light of a particular wavelength, the result is called the Letts effect in this paper, in spite 
of other studies having reported use of a $\mathrm{XeCl}$ excimer laser ${ }^{2,3,4,5,6,7}$ to generate transmutation products rather than heat. The difference between these various laser studies is that the Letts effect implies a much larger nuclear reaction rate if heat is generated by a nuclear reaction, a possibility yet to be determined.

This study has replicated the Letts effect using two different calorimeters and several different substrate materials used as the cathode. The laser effect demonstrates production of anomalous energy without allowing rejection based on proposed errors in calorimetry because relative power is measured over a short span of time, before a change in calibration can take place. In addition, the P-F effect is replicated once again. The two effects are related in that both are sensitive to the nature of material deposited on the cathode surface, but not to the kind of material on which this deposit forms. The two effects appear to be independent of each other, appearing at the same time and as well as separately. In addition, a domain that ceases to be sensitive to the laser does not become spontaneously active and vise versa. However, Fig. 13 shows an example of the Letts effect growing in while the P-F effect disappears.

Although changing the wavelength over the available range appeared to have no effect, certain other wavelengths are expected to have a much different effect.

Because the effects are sensitive to the deposited surface, the nature of these surfaces needs to be explored. However, the deposited layer changes continuously during electrolysis, thereby making a comparison between surface properties measured after the study and behavior during the study rather difficult. In this study, surfaces are deposited

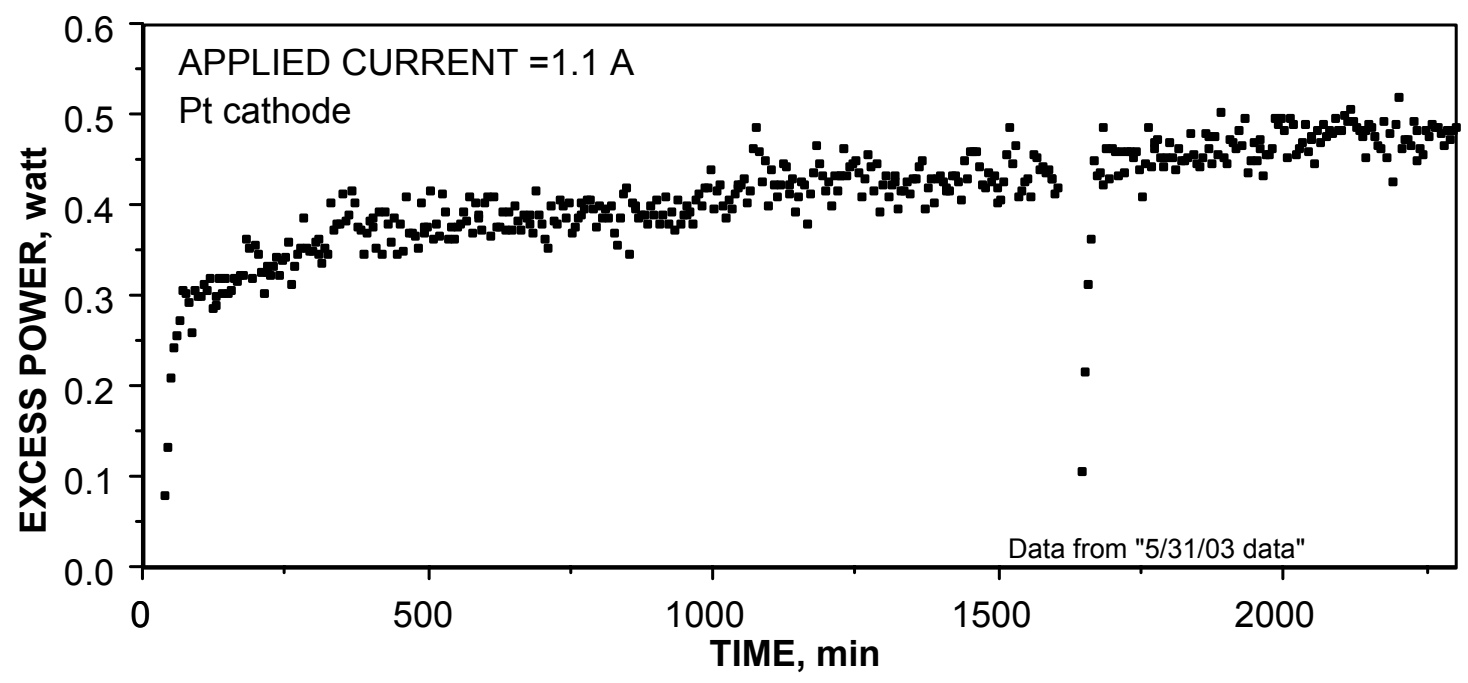

FIGURE 11. Effect of time on excess power while $\mathrm{Pt} \# 2$ cathode is exposed to $1.1 \mathrm{~A}$ of applied current. The cathode is at $47^{\circ} \mathrm{C}$. 


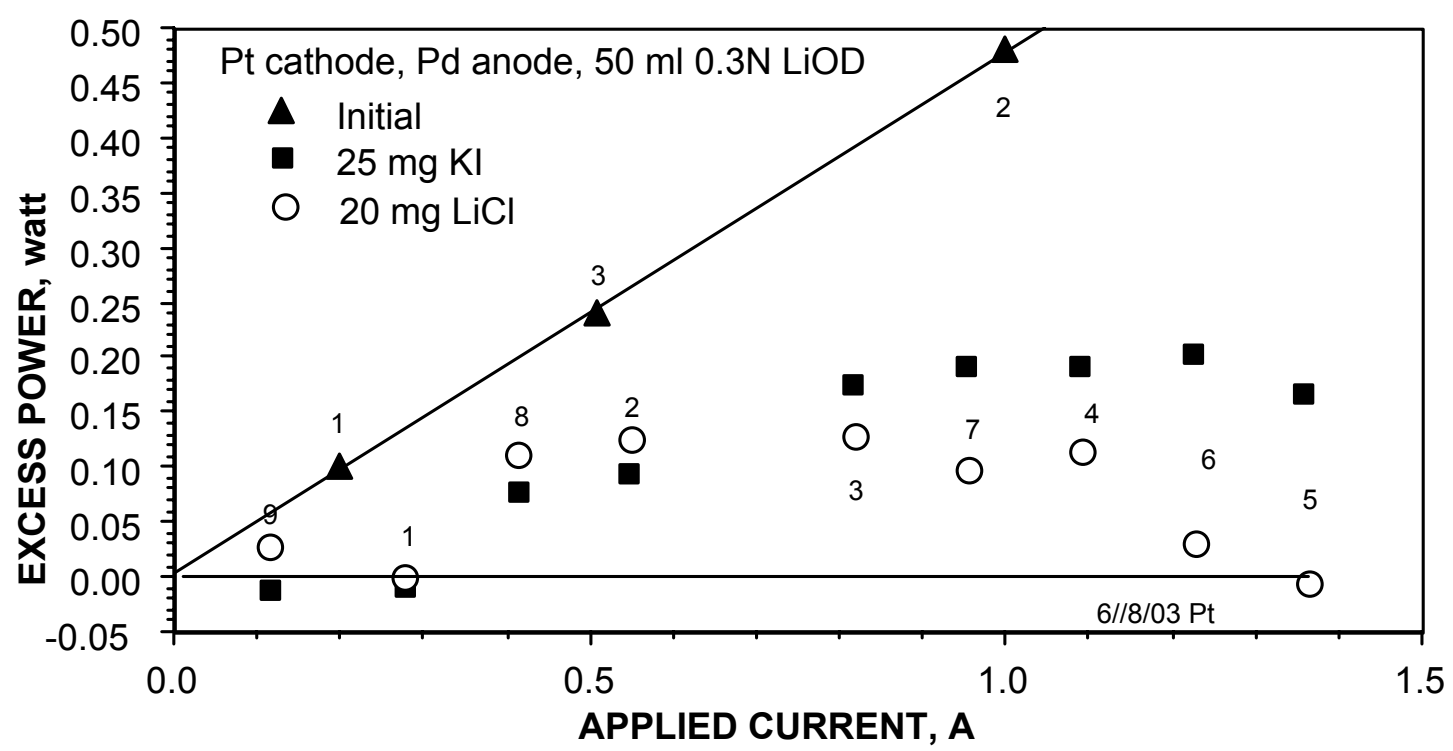

FIGURE 12. Effect of $\mathrm{KI}$ and $\mathrm{LiCl}$ on production of excess energy from a Pt cathode. The sequence with which points are taken is shown. Notice that a critical onset current of about 0.35 $\mathrm{A}$ is required after the material is added in contrast to no onset before.

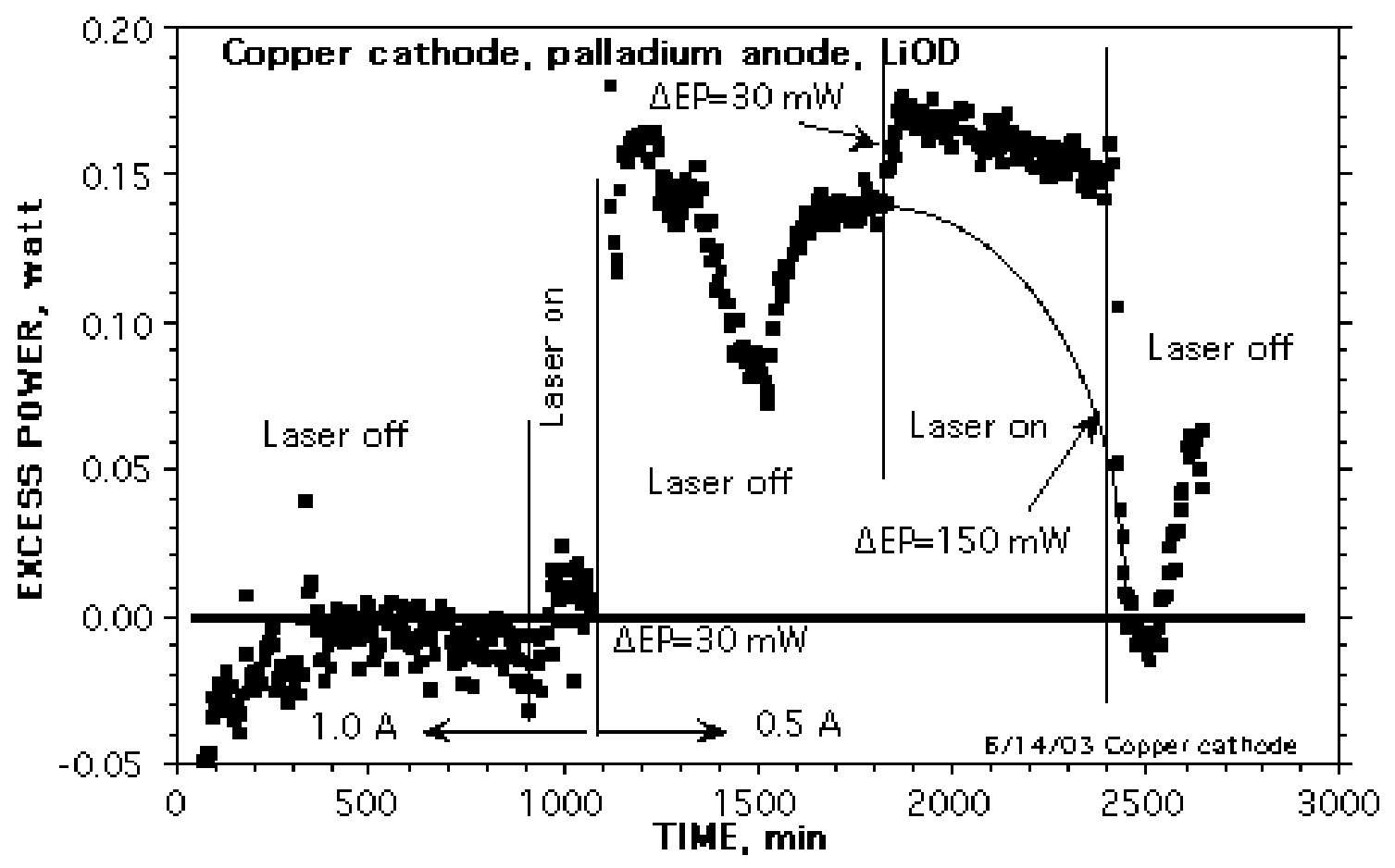

FIGURE 13. Copper cathode electrolyzed at $0.5 \mathrm{~A}$ and $1.0 \mathrm{~A}$ after calibration. The line drawn between 1830 and 2450 min shows how the proposed P-F power changed with time.

outside the cell using various plating solutions and inside the cell by relying on transfer of material from a Pd or Au anode to the cathode.

A few examples of previous work show that platinum can transfer from a platinum anode to a platinum cathode $\mathrm{e}^{8,9,10}$ and cause the cathode to generate $\mathrm{EP}^{11}$. However, this transfer is very 
slow. This study explores the much faster transfer of palladium from a palladium anode to a cathode of various metals. This co-deposition method is similar to, but slower than that used by Szpak et al. ${ }^{12}$ when palladium was supplied by $\mathrm{PdCl}_{2}$ dissolved in the electrolyte. Both methods produce excess power relatively quickly and with complete reproducibility. It is perhaps ironic that if the anode and cathode had been reversed in past studies of the P-F effect, much of the difficulty in replicating the effects would have been eliminated.

Presence of small amounts of iodide or chloride ion reduces the P-F effect. Both of these ions accelerate transfer of material from the anode to the cathode. In addition, increased current, which also increases the palladium deposition rate, also sometimes reduces excess energy production. A faster transfer rate can change conditions on the cathode surface, for example by changing the $\mathrm{Pd} / \mathrm{Li}$ ratio or the morphology of the deposit. The appearance of a critical onset current indicates the presence of sufficient material on the surface to allow an additional loss of deuterium. This loss has to be offset by production of extra deuterium gas at the surface before the critical composition can be achieved. Indeed, parts of the deposit are sometimes found to have fallen off and appear as black flakes on the bottom of the cell. Such poorly attached material would require additional current to achieve the required $\mathrm{D} / \mathrm{Pd}$ ratio.

Speculations can be made about the relationship between the Letts and the P-F effects. Material, in this case palladium, is proposed to deposit on the cathode surface and nanodomains are thought to gradually form in which the anomalous heat producing reaction occurs. These regions either acquire a size and/or composition to make them spontaneously active (P-F effect), or they can have a size/composition that allows application of laser energy to boast them into the energy-producing mode (Letts effect). The greater the number of photons applied to the surface, the greater the amount of anomalous energy, until the process becomes saturated. As additional material is deposited, both effects can either increase or decrease, independent of each other.

The magnitude of the Letts effect is proportional to the number of suitable domains within the area of the laser spot (about $1 \mathrm{~mm}^{2}$ ). Because the active surface is very thin, the Letts effect can generate an effective power density near $100 \mathrm{~kW} / \mathrm{cm}^{3}$, much greater than has been claimed based on the entire volume of the cathode. On the other hand, the magnitude of the P-F effect is proportional to the number of active domains on the cathode surface (about $1.5 \mathrm{~cm}^{2}$ ). If the laser had irradiated the entire surface, the magnitude of its effect would have been approximately 150 times the observed power production. Yet, when the Letts effect disappears and is replaced by the P-F effect, the amount of P-F heat is much less than 150 times the Letts heat. Changes in the surface that result in a loss of Letts effect cause, at the same time, a reduction in the P-F effect. In other words, domains capable of producing the Letts effect do not immediately convert to being spontaneously active domains as their characteristics change. Apparently, some active domains require laser stimulation to generate their maximum energy.

If this explanation is correct, the Letts effect can be powerful tool to investigate the P-F effect and an easy way to demonstrate anomalous energy using even a simple calorimeter. An exploration of other laser wavelengths is expected to reveal values that are able to stimulate energy production from a wider range of conditions than those stimulated by the wavelength used here.

Deposition of a dendritic structure, which is proposed to be one of the active morphologies, is encouraged by low current and low concentration of the deposited ion in the solution. These conditions are typically used during conventional cold fusion studies. In this case, platinum is the deposited material, which has a low concentration in the electrolyte. Because current is kept low at first to prevent cracking of the palladium cathode, dendrite 
formation is encouraged. Thus, without knowing the reason, such studies created ideal conditions to produce the actual active structure on the surface of the cathode. Because palladium is transparent to deuterium, the bulk must be fully loaded to achieve a high composition at the surface. Use of platinum or copper as the substrate avoids this problem because these metals have a small transport rate for deuterium. Deposition of gold on palladium shows an especially large Letts effect as was found in this work and by Dennis Letts in his study. However, the magnitude of the P-F effect is not known for a Pd substrate during either study. In addition, both effects occur when materials are applied to a $\mathrm{Pt}$ or $\mathrm{Cu}$ surface. Neither effect depends only on gold deposited on palladium to work. Theory must now take this additional behavior into account.

\section{REFERENCES}

${ }^{1}$ Letts, D., Laser Initiated Heat Release from Electrolytic Systems, American Physical Society Conference, Austin Convention Center, TX, March 3-7, 2003.

${ }^{2}$ Beltyukov, I.L., et al., Laser-induced cold nuclear fusion in $\mathrm{Ti}_{-}-\mathrm{H}_{2}-\mathrm{D}_{2}-\mathrm{T}_{2}$ compositions, Fusion Technol., 1991. 20: p. 234.

${ }^{3}$ Castellano, et al. Nuclear Transmutation in Deuterated Pd Films Irradiated by an UV Laser, in 8th International Conference on Cold Fusion. 2000. Lerici (La Spezia), Italy: Italian Physical Society, Bologna, Italy.

${ }^{4}$ Nassisi, V., Transmutation of elements in saturated palladium hydrides by an XeCl excimer laser, Fusion Technol., 1998. 33: p. 468.

${ }^{5}$ Nassisi, V. and M.L. Longo, Experimental results of transmutation of elements observed in etched palladium samples by an excimer laser, Fusion Technol. 2000, 37: p. 247. 6 Nassissi, V., Incandescent Pd and Anomalous Distribution of Elements in Deuterated Samples Processed by an Excimer Laser, J. New Energy, 1997. 2(3/4): p. 14.

${ }^{7}$ Steinert, C., Laser-induced 'semicold' fusion, Fusion Technol., 1990. 17: p. 206.

${ }^{8}$ Lihn, C.J., et al., The influence of deposits on palladium cathodes in D2O electrolysis. Fusion Technol., 1993. 24: p. 324.

${ }^{9}$ Ulman, M., et al., Surface and electrochemical characterization of Pd cathodes after prolonged charging in LiOD $+\mathrm{D}_{2} \mathrm{O}$ solutions. J. Electroanal. Chem., 1990. 286: p. 257.

${ }^{10}$ Bockris, J.O.M. and Z. Minevski, Two zones of "Impurities" observed after prolonged electrolysis of deuterium on palladium. Infinite Energy, 1996. 1(5/6): p. 67.

${ }^{11}$ Storms, E. Excess Power Production from Platinum Cathodes Using the Pons Fleischmann Effect. in 8th International Conference on Cold Fusion. 2000. Lerici (La Spezia), Italy: Italian Physical Society, Bologna, Italy.

${ }^{12}$ Szpak, S., P.A. Mosier-Boss, and M.H. Miles, Calorimetry of the Pd+D codeposition Fusion Technol., 1999. 36: p. 234. 